\title{
The Threshold Conditions in Electrical Excitation of the Nerve Fiber. Part I
}

By

\author{
Ichiji Tasaki \\ Department of Medicine, Keio University, Tokugawa Institute \\ of Biological Research
}

Received July 21, 1949

In 1939, Bernhard Katz wrote a comprehensive review of the earlier investigations dealing with the problem of electric excitation of the nerve. Soon after this review was published, however, the method of exciting a single medullated nerve fiber on a "bridge-insulator" began to yield some interesting results in this field. Encouraged by these results, extensive research has since been devoted to the formulation of the threshold conditions in the isolated medullated nerve fiber.

The aim of this article is to help review, by rewriting several chapters of a monograph (Tasaki, 1944) written in Japanese, what kind of experi ments have been done on single fiber preparations and what conclusions have already been drawn from the results obtained. It is not intended to put forward here any speculative theory as to the physico-chemical nature of the excitation phenomena. The content of this article may be regarded as a purely formal, quantitative description of the experimental data.

Problem of nervous transmission remain outside the scope of this article. Furthermore, limitation imposed upon the length of the article has made it practically impossible to discuss in any detail the obvious merits and deficiencies, revealed by the findings described in this article, of the excitation theories advanced by previous investigators.

\section{Excitation by brief voltage pulses}

1.1 The excitatory state. It has been established by Fabre and his coworkers (1933-34) that, with extremely brief electric shocks, the minimum quantity of electricity required for excitation is independent of the form of the shock. This constant quantity relation is obeyed extremely accurately by the isolated medullated nerve fiber.

For such extremely brief shocks, the excitatory effect set up by a subthreshold shock of a quantity $q$ may be expressed by

$$
\frac{q}{\epsilon} \Theta(t)
$$

$\epsilon$ being the minimum, or threshold, quantity stated above and $\Theta(t)$ the function of time which should be determined so as to reconcile with the latent addition phenomena. ${ }^{1}$ With a shock of suprathreshold strength,

1 In this article. Greek letters stand for quantities of functions which depend only on the state of the excitable tissue under investigation. 
namely when $q>\epsilon$, the excitatory effect must rise above a certain definite critical level required to release a nerve impulse.

When two subthreshold brief shocks of quantities $q_{1}$ and $q_{2}$ are delivered at time-interval $z$, the excitatory effects set up these individual shocks are considered to sum. The simplest assumtion as to the law of summation of this type would be to describe the summed excitatory effect by the formula

$$
\frac{q_{1}}{\epsilon} \boldsymbol{\Theta}(t)+\frac{q_{2}}{\epsilon} \boldsymbol{\Theta}(t-z)
$$

In order that this pair of shocks can release an impulse, the summed excitatory effect must rise above the critical level $\nu$ at some moment; viz.,

$$
\operatorname{Max}\left\{\frac{q_{1}}{\epsilon} \Theta(t)+\frac{q_{2}}{\epsilon} \Theta(t-z)\right\} \geqq \nu .
$$

The symbol "Max" is used to describe the maximum value of the function of time $t$ in the parenthesis.

We shall now denote $\Theta(t) / \nu$ by $\mathscr{D}(t)$, then eq. (1), describing the condition which two brief shocks must fulfil in order to set up an impulse, reduces to

$$
\operatorname{Max}\left\{\frac{q_{1}}{\epsilon} \varphi(t)+\frac{q_{2}}{\epsilon} \Phi(t-z)\right\} \geqq 1 .
$$

In the treatment of the experimental data, we are concerned only with the relationship among the variables $q_{1}, q_{2}$ and $z$. We can determine neither the absolute magnitude nor the dimension of the excitatory process itself. For a description of the experimental data, therefore, it is perfectly satisfactory to adopt eq. (2) instead of (1). Any change in the state of the nerve fiber can fully be described by the changes in the

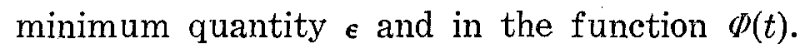

We may now define the dimensionless quantity $\frac{q_{1}}{\epsilon} \phi(t)$ the excitatory state (e.s.) set up by the shock of quanitity $q_{1}$ in the nerve fiber. When $q_{1}=\epsilon$, an impulse is released without accompaniment of the second shock. It is therefore obvious that

$$
\operatorname{Max}\{\tilde{\varphi}(t)\}=1 .
$$

1.2 Experimental determination of the function $\Phi(t)$ We will now proceed to determine the form of the function $\Phi(t)$. It is obvious from the onset that e.s. is zero before and long after delivery of the shock. It must therefore reach the maximum at a certain definite moment $t=a(\geqq 0)$. From eq. (3) it follows that

$$
\Phi(\alpha)=1 \text {. }
$$

When the quantity of the first shock $q_{1}$ is far below the threshold $\epsilon$, an impulse is released only when the quantity of the second shock $q_{2}$ is near the threshold. Under such circumstances, the threshold condition 
(e.s. $=1$ ) is fulfilled approximately at the moment $t=z+\alpha$, when the second term in the parenthesis of eq. (2) reaches the maximum. Hence, introducing $t=z+\alpha$ into eq. (2) and taking the relation of eq. $\left(3^{\prime}\right)$ into consideration, we find that, in threshold excitation, ${ }^{1}$

$$
\frac{q_{1}}{\epsilon} \emptyset(z+\alpha)+\frac{q_{2}}{\epsilon}=1,\left(q_{1} \ll \epsilon, q_{2} \doteqdot \epsilon\right) .
$$

We shall now compare this equation with a set of curves obtained by actual threshold determinations.

Fig. 1 shows the observed relations among these variables obtained from a single nerve fiber preparation. In this figure, the value of $\left(1-q_{2} / \epsilon\right)$ is plotted as ordinate against the time-interval $(z)$ from the shock $q_{1}$ to $q_{2}$. The results for different continuous curves. The accuracy with which threshold determination can be done on single fiber preparations is about 3 per cent or less.

As expected from eq. (4), the curve relating $\left(1-q_{2} / \epsilon\right)$ and $z$ for a given value of $q_{1}$ attains the maximum (or the minimum when $q_{1}$ is negative) at the moment $z=0$, its maximum value being equal to $q_{1} / \varepsilon$. At any interval, $\left(1-q_{2} / \epsilon\right)$ was actually found to be directly proportional ${ }^{2}$ to the

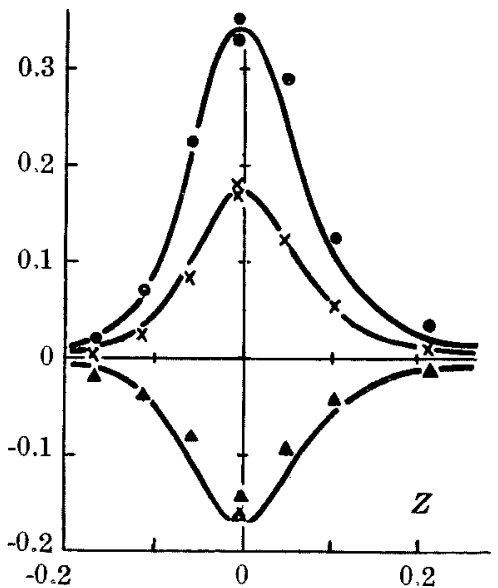

Fig. 1. Time course of threshold reduction caused by a conditioning shock of the strength of $-0.17,0.17$ and 0.34 times the threshold. A toad's motor nerve fiber at $16^{\circ} \mathrm{C}$. Both conditioning and test shocks were rectangular voltage pulses of 0.025 msec. in duration. quantity $q_{\mathbf{l}}$, provided that $q_{1}$ does not exceed about half the threshold (see also Fig. 6, right).

2.3 Threshold reduction caused by a series of brief shocks. Let us now consider the case in which a series of brief shocks, carrying quantities $q_{0}, q_{1}, q_{2}, \ldots$, are delivered in succession. Then, the excitatory state

I A closer analysis, in which the shift of the maximum in the superposed curve is taken into consideration, indicates that, for $\left|q_{1}\right| \ll \varepsilon$,

$$
\varepsilon-q_{2}=q_{\mathrm{I}} \Phi(z+\alpha)+\frac{-q_{\mathrm{r}}{ }^{2}\left\{\Phi^{\prime}(z+\alpha)\right\}^{2}}{q_{2} \Phi^{\prime \prime}(\alpha)+q_{1} \Phi^{\prime \prime}(\alpha+Z)}
$$

where $\Phi^{\prime}(t)$ ard $\theta^{\prime \prime}(t)$ represent the first and second derivatives of the function respectively. As the denominator in the last term is of a negative value, this small correction term is always positive.

2 A closer analytical treatment of the observed data reveals that there is slight but systematic deviation of the observed values from this proportionality. Introduction of the observed curve of Fig. 3 , left, into the function $\varphi(t)$ in the correction term stated in the preceding foot-note, tells us that, for $q_{1} / \varepsilon=0.5$, this term should amount approximately to 6 per cent and, $q_{1} / \varepsilon=-0.5$, to +3 per cent (both at $z=0.5 \mathrm{msec}$.). The observed deviation from the proportionality could fully be interpreted as resulting from the neglect of the correction term stated above. 
$E_{l l}(t)$ set up by these shocks in the nerve fiber should be given, according to our assumption of superposition of e.s., by the formula

$$
E_{b}(t)=\frac{q_{0}}{\epsilon} \Phi(t)+\frac{q_{1}}{\epsilon} \Phi\left(t-z_{1}\right)+\frac{q_{2}}{\epsilon} \phi\left(t-z_{2}\right)+\ldots,
$$

where $z_{i 2}$ denotes the interval from the zero-th shock to the $n$-th.

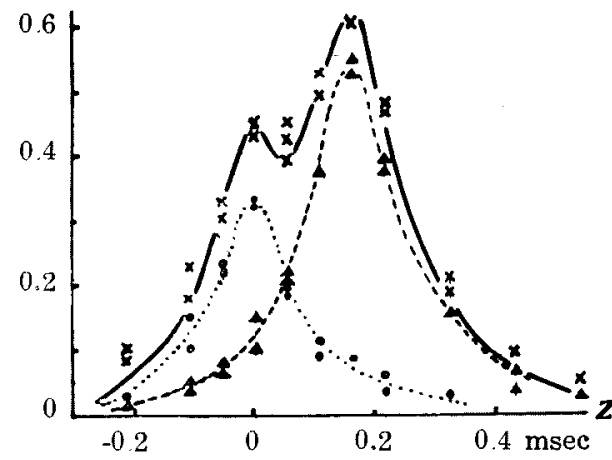

Fig. 2. Solid line: Threshold reduction caused by two shocks, the first 0.32 times and the second 0.52 times the threshold strength, delivered at interval of 0.162 msec. Broken and dotted lines: Threshold reduction by these indiuidual subthreshold shocks. $16^{\circ} \mathrm{C}$. (TASAKI, 1942 .
If all these shocks are far below the threshold $\epsilon$, the time course of $E_{l}(t)$ should be determined by adding another, stronger shock which is generally termed the "test-stock". Let the time-interval from the zero-th shock to the test shock, of quantity $S$, be denoted by $z$, then the maximum of the composite e.s. is attained at approximately $t=z+\alpha$ when e.s. caused by the test shock alone reaches the maximum. Under these conditions, the formula describing the condition of threshold determination by the shock test method, $\operatorname{Max} E_{b}(t)=1$, reduces to

$$
\frac{q_{0}}{\epsilon} \Phi(z+\alpha)+\frac{q_{1}}{\epsilon} \Phi\left(z+\alpha-z_{1}\right)+\frac{q_{2}}{\epsilon} \Phi\left(z+\alpha-z_{2}\right)+\ldots=1-\frac{S}{\epsilon} .
$$

Now, it should be emphasized that the quantity $(1-S / \epsilon)$, which we may for simplicity call the "threshold reduction ", 1 does not give us the form of $E_{l}(t)$ of eq. (5), but it gives us the time course of $E_{l}(z+\alpha)$. Unless actual experimental data proves that $\alpha=0$, the curve showing the time course of the threshold reduction (a function of the interval $z$ ) precedes the time course of e.s. by the time $\alpha$, which is required for the function $\Phi(t)$ to attain the maximum.

Actual measurements of the threshold reduction set up by two or three successive subthreshold shocks indicated very clearly that the relation of eq. (6) is very accurately obeyed (Fig. 2). The form of the function $\Phi(z+a)$ can be determined by the method described in the preceding section. Using this function, the sum on the left-hand side of eq. (6) can easily be calculated and the result of calculation can be compared with the actual data. The agreement between the observed and calculated values was perfectly satisfactory.

1.4 The form of the function $\Phi(t)$ The function $\varphi(t+\alpha)$ as determined from the threshold reduction curve is of the form as shown in Fig. 3,

1 Katz (1939) gave this quantity a wrong name the "local excitatory disturbance". 
right. In order to obtain the curve for the function $\Phi(t)$, it is necessary to know the absolute value of the constant $\alpha(\geqq 0)$ which depends only on the state of the tissue under investigation. As it is obvious that $\Phi(t)$ must vanish for $t<0$, canstant $\alpha$ must be greater than about 0.25 msec. in the case of Fig. 3.

It is known that, in the threshold excitation with brief shocks, an impulse is released at about $0.4 \mathrm{msec}$. after delivery of the shock (at about $15^{\circ} \mathrm{O}$, Tasaki and Maruhashi). The constant can not be greater that this longest latent period. Hence, at room temperature,

$$
0.25<\alpha<0.4
$$
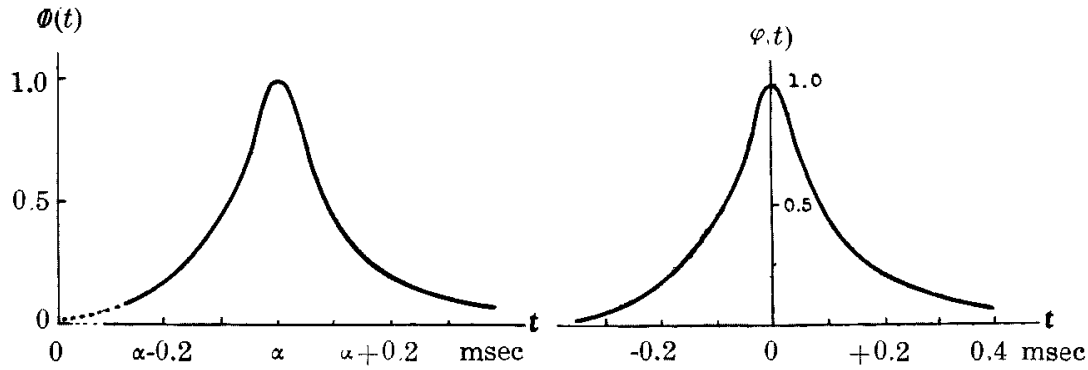

Fig. 3. Forms of the function $\Phi(t)$ and $\phi(t)$ determined on a toad's motor nerve fiber at $16^{\circ} \mathrm{C}$. (TASAKI, 1942).

Inasmuch as we are concerned only with threshold measurements, we have no direct way to determine the value of $\alpha$ more rigidly.

As it is the function $\bar{\varphi}(t+\alpha)$, and not $\Phi(t)$, that is directly determined by threshold measurements, we will introduce a function $\varphi(t)$ by

$$
\varphi(t) \equiv \bar{\varphi}(t+\alpha) .
$$

Introducing this new function into eq. (6) and relacing the variable $z$ with $t$, we obtain

$$
\sum_{i} \frac{q_{i}}{\epsilon} \phi\left(t-z_{i}\right)=1-S / \epsilon,
$$

and this formula is undoubtedly best suited to describe the experimental results of Fig. 2.

1.5 Threshold reduction by a continuous voltage pulse. Let us now calculate the e.s. set up by a continuous voltage $V(t)$. We may regard this voltage as a very rapid succession of brief shocks. The quantity of the elementary shock lasting from $t=z$ to $t=z+d z$ is given by $V(z) d z .^{1}$ The e.s. $d E_{b}$ set up by this elementary shock has, at moment $t$, a magnitude given by

$$
d E_{b}=\frac{V(z) d z}{\epsilon} \Phi(t-z)
$$

1 We express also the minimum quantity $\varepsilon$ simply by the product of the voltage into the duration. In the toad motor nerve fiber, it is generally between 10 and $15 \mathrm{mV} \cdot \mathrm{msec}$. at $15^{\circ} \mathrm{C}$. 
The total e.s. $E_{b}(t)$ set up by all the elementary shocks before the moment $t$ should therefore be given by

$$
E_{t,}(t)=\int_{0}^{t} V(z) \phi(t-z) d z
$$

Introducing into this equation the function $\phi(t)$ which can be determined directly by the experiment of Fig. 1, we obtain instead of (10) the relation

$$
E_{h}(t+a)=\int_{0}^{t+a} \frac{V(z)}{\epsilon} \varphi(l-z) d z
$$

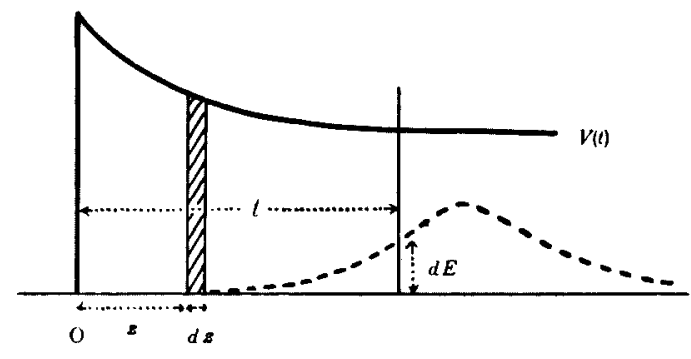

Fig. 4. Diagram illustrating the parameter $z$ in eqs. (9) to (12)

A test shock delivered at moment $t$ in Fig. 4 produces e. s. which attains the maximum at the time $t+\alpha$. When, therefore, the continuous voltage $V(t)$ is far below the threshold, we can determine the magnitude of $E_{b}(t+a)$ in (11) by applying a test shock at the moment $t$. Hence, denoting the threshold measured at this moment by $S$, we have

$$
1-S / \epsilon=\int_{0}^{t+\alpha} \frac{V(z)}{\epsilon} \varphi(t-z) d z
$$

This equation can be derived more directly from the relation of (8).

An extensive test of what is predicted by this equation has been carried out with single fiber preparations. The minimum quantity $\epsilon$, the function $p(t)$ and the threshold reduction by a subthreshold constant voltage were determined on one and the same preparation. And the values calculated by eq. (12) for $V(t)=v$ were compared with the observed values (Tasaki, 1942; 1949). Agreement between the calculated and observed values was very satisfactory.

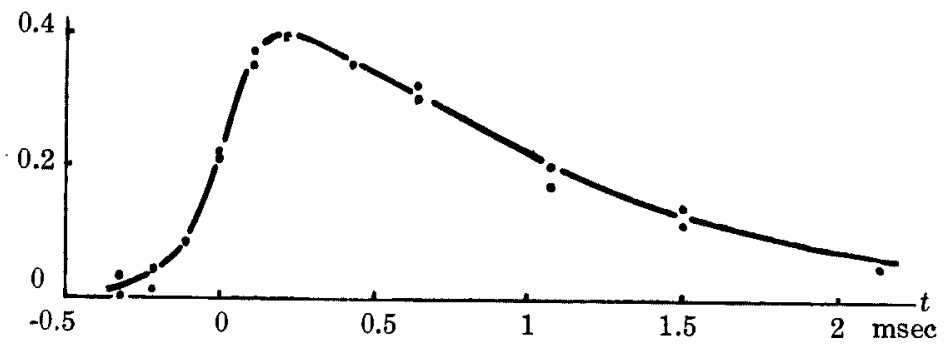

Fig. 5. Dots: Threshold reduction caused by a subthreshold condenser discharge of an initial voltage of $20 \mathrm{mV}$ and of a duration $(r c)$ of $1 \mathrm{msec}$. Continuous line: The curve obtained by calculation for this case. $15^{\circ} \mathrm{C}$. (Tasaki, 1942). 
The dots in Fig. 5 show the observed values of threshold reduction set up by a condenser shock of the time constant of 1 msec. The continuous line in the figure represents the values obtained by calculation. The value of $\epsilon$ and the function $\phi(t)$ used in the calculation were obtained by direct observation on the same preparation.

Emphasis should be laid in these experiments on the fact that the curve showing the time course of threshold reduction extends to the negative side of the time axis (see Fig. 5 and 9). This can also be seen in the data obtained previously by Erlanger and Blair (1931) and is a logical consequence of the delayed maiximum of the function $\Phi(t)$.

1.6 Excitation by two brief shocks. In 1935, Erlanger and Blair discovered that, in excitation of the nerve by two brief shocks, the threshold condition can be expressed by an equation of the form of (1) or (2) in the previous section (also Blair, 1938). This has been confirmed on isolated single nerve fibers. Fig. 6 shows an example of the results obtained.

In this example, the function $\phi(t)$ had a form as shown in Fig. 6, left. Two brief shocks, both about $0.025 \mathrm{msec}$. in duration, were fixed at interval of $0.054 \mathrm{msec}$. and the strengths of these shocks were so controlled that a nerve impulse could be just released by these two shocks. The result of such threshold determination was plotted in Fig. 6 , right.

We can naturally derive the result of Fig. 6 , right, from the function $\varphi(t)$ in this preparation. Using $\phi(i)$ in this preparation. Using $\phi(t)$ instead of $\phi(t)$, eq. (2) becomes in this case
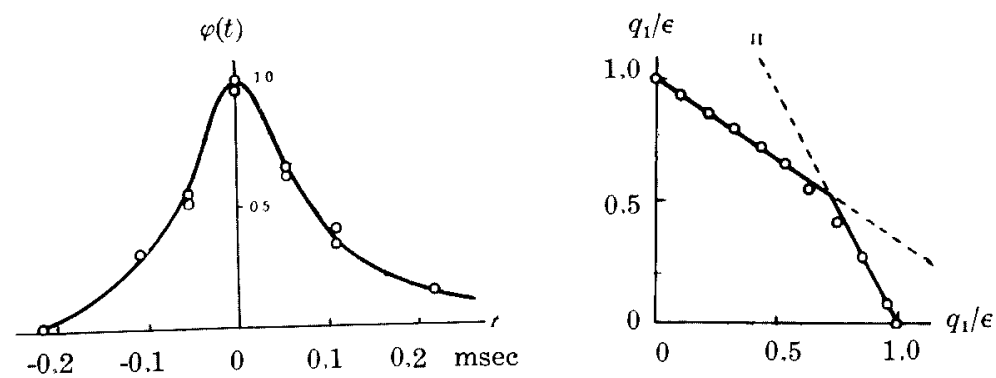

Fig. 6. Left: Relation between strengths of two brief shocks, delivered at an interval of $0.045 \mathrm{msec}$., which just excited a single nerve fiber of the toad. Right: The form of $\varphi(t)$ in this preparation. $13.5^{\circ} \mathrm{C}$. (Tasaki, 1944, p. 185).

$$
\operatorname{Max}\left\{\frac{q_{1}}{\epsilon} \varphi(t)+\frac{q_{2}}{\epsilon}(t-0.054)\right\}=1 .
$$

In case where $q_{1} \ll \epsilon$ and, as a consequence, $q_{2} \doteqdot \epsilon$, the maximum of the function in the parenthesis is attained, as shown in Fig. 7, right, at approximately $t=0.054$. As $\phi(\alpha)=\phi(0)=1$ and, from the curve in Fig. 6, left, $\varphi(0.054)=0.67$, eq. (13) reduces in this case to 


$$
0.67 q_{1}+q_{2}=\epsilon .
$$

The straight line expressed by this equation is shown by $I$ in Fig. 6, right.

If, on the contrary, $q_{2} \ll \epsilon$ and $q_{1} \div \epsilon$, the maximum of the function in eq. (13) is reached at approximately $t=0$. As $\phi(-0.054)=0.59$ in this preparation, eq. (13) now becomes

$$
q_{1}+0.59 q_{2}=\epsilon,
$$

and this relation is shown by the straight line $I 1$ in the figure.

Observed points lie actually on these two straight lines, except in the intermediate region where the maximum of the function in the parenthesis of eq. (13) is considered to reach the maximum between $t=0$ and 0.054 . We now see very clearly how the threshold for the test shock is reduced by a so-called conditioning shock.
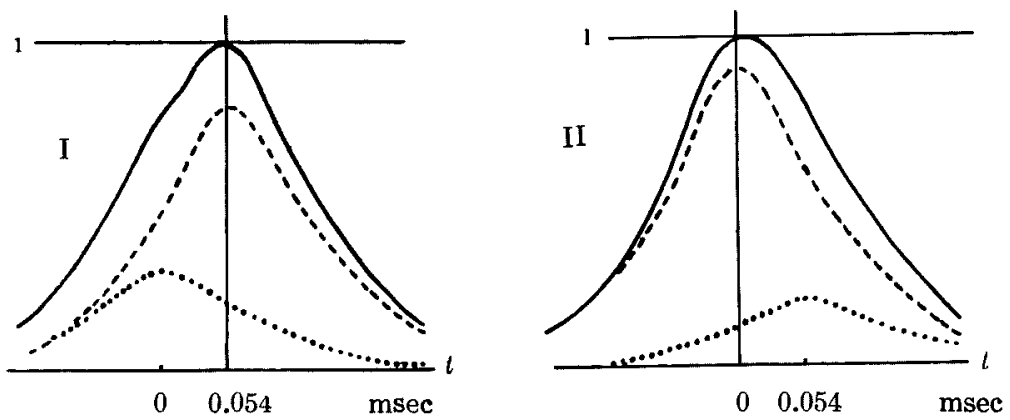

Fig. 7. Diagram illustrating two modes of summation of the e.s. in threshold excitation by two brief shocks. (Tasaki, 1944, p. 186)

1.7 The strength-duration relation. In stimulating a nerve fiber by a continuous voltage pulse $V(t)$, it is expected that excitation occurs when the e.s. set up by this pulse exceeds unity. Eq. (10) or (11) gives the e.s. set up by $V(t)$. Therefore, the equation

$$
\operatorname{Max} \int_{0}^{t+\alpha} \frac{V(z)}{\epsilon} \varphi(t-z) d z=1
$$

is expected to describe the threshold condition for $V(t)$.

In the case of a condenser discharge of the time constant $r c$, $V(t)=v e^{-\tau / n c} ;$ then eq. (14) becomes

$$
v=\varepsilon / \operatorname{Max} \int_{0}^{t+\alpha} e^{-z / r c} \phi(t-z) d z
$$

As the member on the left-hand side of this equation is, for a given preparation, a function of only $r c$, this represents the voltage capacity relation predicted for this case. Calculation of this equation is somewhat tedious, but in a few preparations numerical calculation was actually performed (Tasaki, 1942). For the range $0.015<r c<0.4 \mathrm{msec}$, agreement between the calculated and observed values was perfect. For 
durations longer than about $0.4 \mathrm{msec}$, the observed threshold voltages were found to be constantly smaller than the calculated. We will see in the next chapter that the relation of eq. (14) is the sufficient, but not the necessary condition for releasing an impulse by relatively short voltage pulses.

For a constant voltage of the $(\mathrm{mV})$ magnitude $v$ and the duration $T$, eq. (14) becomes

$v=\epsilon / \operatorname{Max} \int_{0}^{T} \varphi(t-z) d z$.

Numerical calculation of the $v-T$ relation expressed by this equation is fairly simple. Fig. 8 gives an example of the experiments done to test this result. As can be seen in the figure, agreement between the observed and calculated values is satisfactory for voltage pulses of relatively short durations. For duration above about 0.4 msec., however, there is again a systematic divergence between the observed and calculated values.

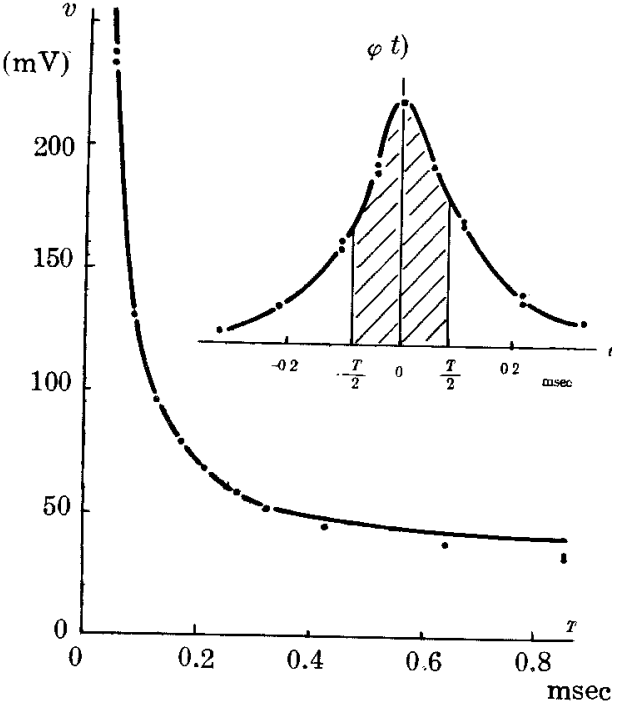

Fig. 8. Strength-duration relation, observed (dots) and calculated (continuous line). $16^{\circ} \mathrm{C}$. (Tasaki).

\section{Superposition of the excitatory state}

2.1 Superimposition of a brief shock to a constant voltage pulse. If one superimpose, at various intervals, a brief shock on a long rectangular voltage pulse, one finds that threshold for the brief shock is distinctly reduced during the passage of the constant current. The change in the threshold is, within a certain definite limit, approximately proportional to the constant voltage (see Fig. 9.) The threshold reaches a steady level within a fraction of millisecond and this level is maintained for a long period after the make of the constant voltage, provided that the constant voltage is less than about half the rheobase. ${ }^{1}$

If one fix the interval $t$ and adjust the strengths $v$ and $q$ so that a nerve impulse is barely released by this compound stimulus, one obtains a diagram as shown in Fig. 9, right. In this diagram the observed points near the two axes can be regarded as lying on straight lines $\mathrm{I}^{2}$ and II. In

1 If the constant voltage is nearly rheobasic, the threshold for the brief shock shows a gradual increase after a minimum is reached, as stated by Erlanger and Blair (1981), Rushton (1932) and others.

2 According to eq. (12), straight line $I$ is given by

$$
1-S / \varepsilon=v(1 / \varepsilon) \int_{0}^{\infty} \varphi(Z) d z
$$


the intermediate region, observed points are connected by a smooth curve.

Attention should be called in this experiment that, no matter what the interval is, all the observed points do not lie on a single straight line. The fact that the straight line II is almost perpendicular to the abscissa was interpreted by Rushton (1937) and Katz (1939) as indicating the presence of a nonpropagated impulse at the site of stimulation. The interpretation which we are going to give to these data is, at least by appearance, different from that given by the british investigators.

We interpret the straight line I to indicate the contribution of the constant voltage to the e.s. set up by the brief shock. It is obvious that, in case where $v=0$, the threshold condition is fulfilled by the
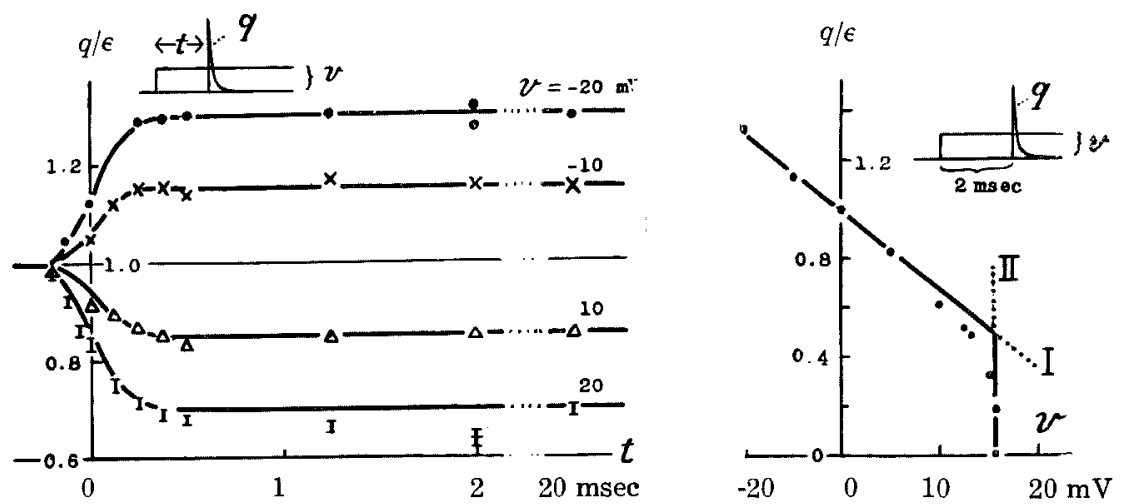

Fig. 9. Left: Relation between threshold strength for an induction shock (ordinate) and time from the make of long constant voltage pulses of four different magnitudes (abscissa). Right: Relation between constant voltage and threshold strength for an induction shock superimposed as shown in the diagram. Toad's motor nerve fiber at $7^{\circ} \mathrm{C}$. The induction shock decays exponentially at a time constant of $0.02 \mathrm{msec}$.

developement of e.s. set up by the brief shock. The reduction of this threshold level by addition of a constant voltage should therefore be attributed to an increase of "this" e.s. by addition of the constant voltage. The linearity of the $q-v$ relation in Fig. 9, right, indicates that the contribution of the constant voltage to "this" e.s. increases directly with increasing voltage, and this point has been stressed in the preceding chapter.

In an entirely analogous manner, the straight line II is to be interpreted as representing contribution of the brief shock to "that" e.s. set up by the constant voltage. This contribution is considered to be very small as straight line II is almost parallel to the vertical axis.

2.2 Superimposition of two rectangular voltage pulses of different durations. If one adjust the strengths of two rectangular voltage pulses of different durations, applied to a nerve fiber at a definite time relation, in such a manner that this composite stimulus is just, and only just, effective to excite the fiber, one can figure out how the excitatory 
processes set up by these individual pulses are added at the plasma membrane. An addition of this type can be illustrated by a diagram in which one of the variables representing the strengths is plotted against the other.

In such a diagram, the observed relation between the two variables $v$ and $v^{\prime}$ are represented generally by two or three straight lines. In the example furnished in Fig. 10, the observed points relating $v$ and $v^{\prime}$ which fulfil the threshold condition lay on two straight lines. The durations and the relative position of these two pulses are illustrated by a diagram. Oscillograph records attatched to this figure are those taken at the site of stimulation when these pulses were applied to the fiber.

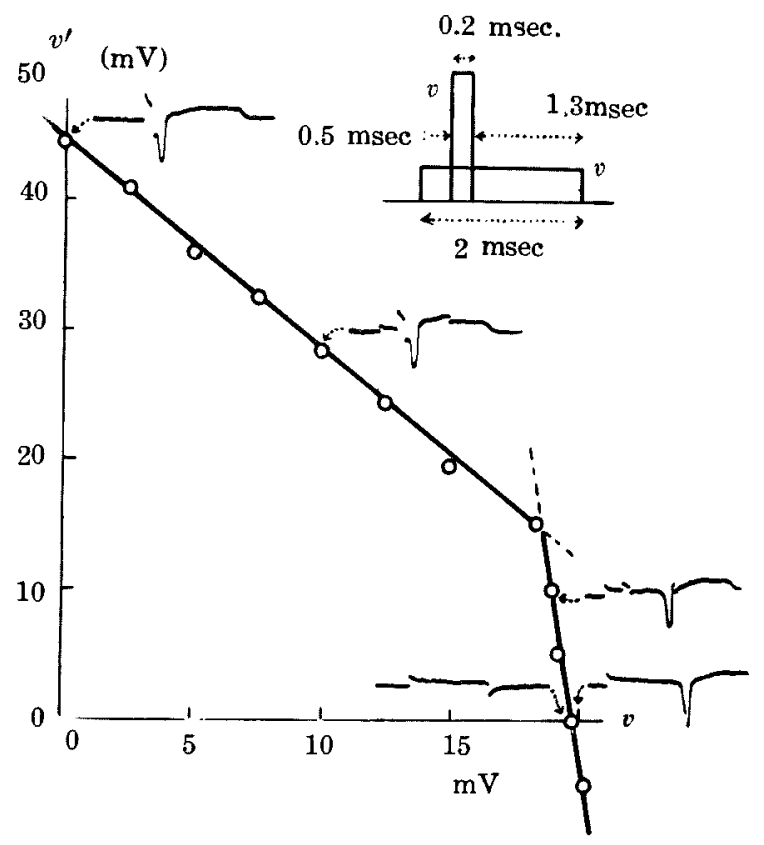

Fig. 10. Relation between strengths of two superimposed voltage pulses which barely excite a motor nerve fiber of the toad. Osciliograph records were obtained with the stimulating indicated by the arrows in the figure. $16^{\circ} \mathrm{C}$. (Tasaki, 1944, p. 156).

In this example, it is seen that, inasmuch as the points representing pairs of $v$ and $v^{\prime}$ lie on one and the same straight line, change of the ratio of $v$ to $v^{\prime}$ does not affect the latent period of action current appreciably. Transition from one straight line to another resulted in an abrupt change in the latent period. With two voltage pulses of shorter durations, such an abrupt change in the latent period was not very distinct.

These findings appeared at first sight to indicate that, at least in the experiment of Fig. 10, the e.s. set up by the composite stimulus reaches the maximum at two different moment during its course and that the bend of the $v-v^{\prime}$ relation into two or more straight parts resulted 
solely from the difference in the time at which the e.s. attains the maximum. But, the following observation rendered this view untenable.

If the difference in the crest time of the e.s. were the only cause for the bend in the $v-v^{\prime}$ relation, we should be able to take such a bend away by choosing an appropriate relative position of these two pulses. Actually, in the experiment of Fig. 10, it was found that, no matter what the interval is, the bend in the $v-v^{\prime}$ relation never disappears. Many other experiments done to test this point indicated very clearly that unless both of the two pulses are of the duration shorter than about $0.3 \mathrm{msec}$. or longer than about 3 msec., the $v-v^{\prime}$ relation can not be represented by a single straight line, regardless of the relative position of the two pulses. We have seen already that the excitatory effect of an extremely brief shock can not be superposed upon that of a very long pulse in full.

2.3 Two extreme cases. When both of the two rectangular voltage pulses are of the durations shorter than about $0.3 \mathrm{msec}$., it is possible to add the excitatory effects of one pulse to the effect of the other in full. In other words, the relation between the strengths $v$ and $v^{\prime}$ which fulfil the threshold condition can be represented by a single straight line, if the interval between these pulses is properly chosen (Fig. 11).
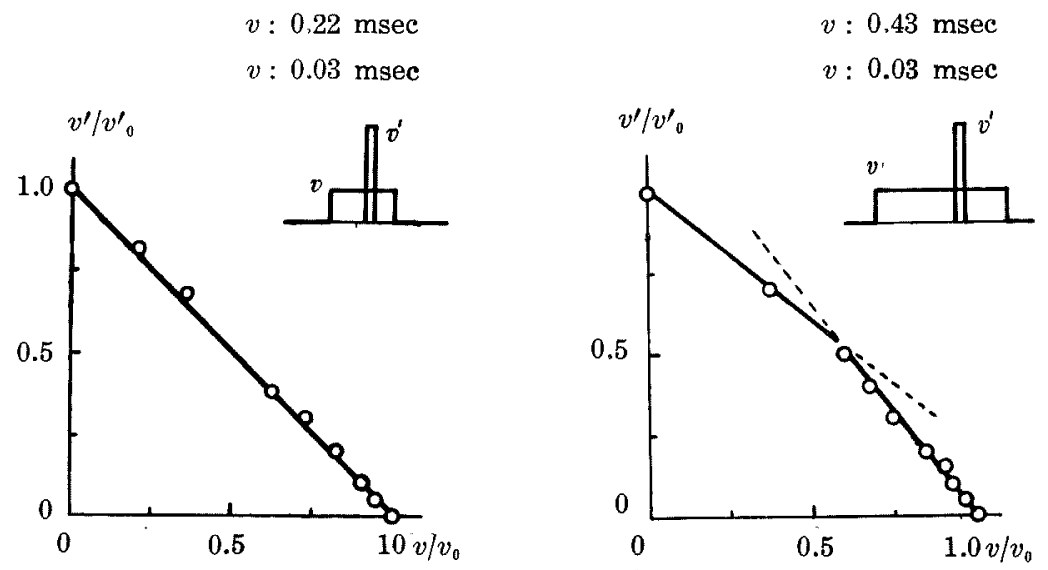

Fig. 11. Relation between strengths of two rectangular voltage pulses superimposed in such a manner that the summation of the excitatory effects was the greatest. Toad's motor fiber at $15^{\circ} \mathrm{C}$. (Tasaki, 1944, p. 165).

But, when one of these pulses is made longer than 0.4 msec., it becomes impossible to represent the observed $v-v^{\prime}$ relation by a single straight line. This critical duration corresponds, it should be pointed out, to the duration at which the observed strength-duration relation begins to deviate from that calculated from function $\varphi(t)$ (Fig. 8).

With two rectangular voltage pulses, both having durations longer than 3 msec. and starting at approximately the same moment, one obtains a very simple $v-v^{\prime}$ relation which can be expressed by a single straight 
line (see Fig. 12, left). It has been shown further that a "triangular" voltage pulse having a relatively long ascending phase superimposed to a long rectangular pulse yield a single straight line (Fig. 12, right).

All these facts suggest very strongly that a nerve impulse is released, not by a single sort of e.s., but many different sorts of e.s. In other words, a rectangular voltage pulse produces many different sorts of e.s. simultaneously at the plasma membrane of the nerve fiber. With brief voltage pulses, a special sort of e.s. which rises and decays swiftly play the role to release an impulse, while with longer pulses a different
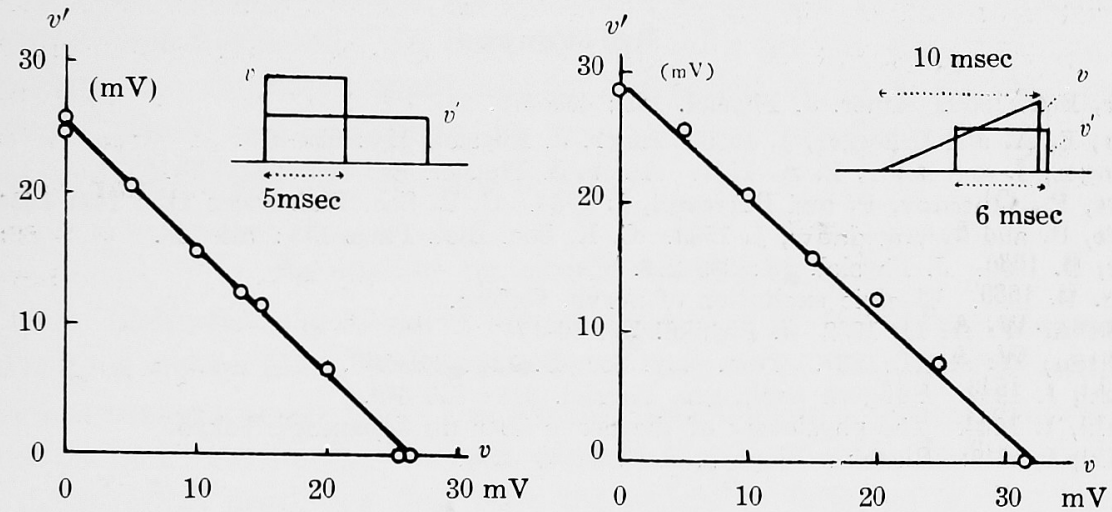

Fig. 12. Relation between the voltage of a rectangular pulse and the strength of another, rectangular or triangular, pulse which barely excited a tord's motor nerve fiber when applied concurrently. $15^{\circ} \mathrm{C}$. (TASAKI, 1944, p. 199).

sort of e.s., which rises more slowly and, consequently, can accummulate more, takes the decisive part. Unless a series of experiments are so designed that only one special sort of e.s. plays a decisive role in all cases, one can not treat those experimental data on the assumption of a simple trigger mechanism.

We have seen in the preceding chapter a series of experiments in which the e.s. $E_{b}$ set up by a brief shock plays a decisive role in releasing impulses. The experimental results of Fig. 12 are a strong indication that, in the experiments dealing with only long, slowly varying voltage pulses, the threshold conditions can be formulated in terms of a single sort of e.s. And, that it is actually the case will be demonstrated in Part II of this article.

\section{Summary}

It was stressed that the excitatory state, which is produced by a stimulating current in the nerve fiber and releases a nerve impulse when it rises above a certain definite critical level, does not decay exponentially, as has been assumed in the previous theories, after removal of the current. A brief stimulating current set up an excitatory state which 
rises first slowly, then quickly and finally reaches a maximum a fraction of a millisecond after termination of the current. After this maximum is reached, it begins to decay (Fig. 4, left).

The time course of the excitatory state was shown to vary, as the course of the stimulating current, in accordance with "the law of proportionality and superposition" (Figs. 1, 2,6 and 9; equation 10).

Evidence was presented to believe that the law of excitation by long, slowly varying stimulating currents is different from that for short stimulating currents.

\section{References}

Blair, E.A. 1938. Amer. J. Physiol. 123 : 455-470.

Blair, E. A. and Erlanger, J. 1936, Amer. J. Physiol. 114 : 309-316.

Erlanger, J. and Blair, E. A. 1931. Amer. J. Physiol. 99 : 108-128; 1298155.

Fabre, P., Quesnoy, P. and Berteaux, J. 1934, C, R. Soc. Biol. Paris 115 : 1431-1434.

Fabre, P. and Swyngedauw, J. 1933 . C. R. Soc. Biol. Paris 113: 765-768.

Katz, B. 1936. J. Physiol. 88 : 239-255.

Ka tz. B. 1939. Electric excitation of nerve. Oxford.

Rushton, W. A. H. 1932 J. Physiol. $75: 16-17$

Rushton, W. A. H. 1937. Proc. Roy. Soc. B. $124: 201-243$.

Tasaki, I. 1942. Pflügers Arch. ges. Physiol. 245 : 665-679.

Tasaki, I. 1944. The physiology of the nerve fiber (in Japanese), Tokyo.

Tasaki, 1. 1949. Biochim. Biophys. Acta 3:49_-509. 